\title{
Hereditary sensory neuropathy type 1 mutations confer dominant negative effects on serine palmitoyltransferase, critical for sphingolipid synthesis
}

\author{
Khemissa Bejaoui, ${ }^{1}$ Yoshikazu Uchida, ${ }^{2}$ Satoshi Yasuda, ${ }^{3}$ Mengfatt Ho, ${ }^{1}$ \\ Masahiro Nishijima, ${ }^{3}$ Robert H. Brown, Jr., ${ }^{1}$ Walter M. Holleran, ${ }^{2,4}$ and Kentaro Hanada ${ }^{3}$ \\ ${ }^{1}$ Day Neuromuscular Research Laboratory, Charlestown, Massachusetts, USA \\ ${ }^{2}$ Dermatology Service and Research Unit, Veterans Affairs Medical Center, Department of Dermatology, School of Medicine, \\ and Department of Pharmaceutical Chemistry, University of California, San Francisco, California, USA \\ ${ }^{3}$ Department of Biochemistry and Cell Biology, National Institute of Infectious Diseases, Tokyo, Japan \\ ${ }^{4}$ School of Pharmacy, University of California, San Francisco, California, USA
}

Hereditary sensory neuropathy type 1 (HSN1) is a dominantly inherited degenerative disorder of the peripheral nerves. HSN1 is clinically and genetically heterogeneous. One form arises from mutations in the gene SPTLC1 encoding long-chain base 1 (LCB1), one of two subunits of serine palmitoyltransferase (SPT), the enzyme catalyzing the initial step of sphingolipid synthesis. We have examined the effects of the mutations $\mathrm{C} 133 \mathrm{Y}$ and $\mathrm{C} 133 \mathrm{~W}$, which we have identified in two HSN1 families, on the function of SPT. Although in HSN1 lymphoblasts, the C133Y and C133W mutations do not alter the steady-state levels of LCB1 and LCB2 subunits, they result in reduced SPT activity and sphingolipid synthesis. Moreover, in a mutant Chinese hamster ovary $(\mathrm{CHO})$ cell strain with defective SPT activity due to a lack of the LCB1 subunit, these mutations impair the ability of the LCB1 subunit to complement the SPT deficiency. Furthermore, the overproduction of either the LCB1C133Y or LCB1C133W subunit inhibits SPT activity in CHO cells despite the presence of wild-type LCB1. In addition, we demonstrate that in CHO cells the mutant LCB1 proteins, similar to the normal LCB1, can interact with the wild-type LCB2 subunit. These results indicate that the HSN1-associated mutations in LCB1 confer dominant negative effects on the SPT enzyme.

J. Clin. Invest. 110:1301-1308 (2002). doi:10.1172/JCI200216450.

\section{Introduction}

Hereditary sensory neuropathy type 1 (HSN1) is a debilitating peripheral neuropathy predominantly affecting the distal lower extremities. In early stages of the disease, pain and temperature sensations are most affected (1). Symptoms begin with loss of sensation in the feet and the hands, progress to ulcers, and result in mutilating, hyperkeratotic lesions of the fingers and toes that often require amputation. As the disease progresses, there is also degeneration of motor neurons with secondary denervation atrophy and weakness of distal limb muscles (2). The mode of inheritance of HSN1 is autosomal dominant, with onset typically in the second or third decade (3).

Received for publication July 18, 2002, and accepted in revised form September 17, 2002.

Address correspondence to: Kentaro Hanada, Department of Biochemistry and Cell Biology, National Institute of Infectious Diseases, 1-23-1, Toyama, Shinjuku-ku, Tokyo 162-8640, Japan. Phone: 81-3-5285-1111; Fax: 81-3-5285-1157; E-mail: hanak@nih.go.jp.

Conflict of interest: No conflict of interest has been declared. Nonstandard abbreviations used: hereditary sensory neuropathy type 1 (HSN1); long-chain base 1 (LCB1); serine palmitoyltransferase (SPT); coenzyme A (CoA);

3-ketodihydrosphingosine (KSD); glucosylceramide (GlcCer); Chinese hamster ovary (CHO); pyridoxal phosphate (PLP); sphingomyelin (SM); ceramide (Cer); phosphatidylserine (PS); PLP-dependent $\alpha$-oxoamine synthase (POAS); 8 -amino-

7-oxononanoate synthase (AONS); dorsal root ganglia (DRG).
HSN1 is a clinically heterogeneous disease. The characteristic features described above may be associated with lancinating pain. Deafness has been reported in some families $(4,5)$. The expression of the disease also varies by gender within the same family. The symptoms begin later in women than in men, and the progression is usually more severe in men (6). In addition to the clinical heterogeneity, HSN1 is characterized by a genetic heterogeneity, since at least three gene variants are reported. The genetic loci for two variants are defined, with linkage of HSN1 to both chromosome 9q22 (7-9) and chromosome 3q13-22 (10). Moreover, linkage to both the $9 \mathrm{q}$ and the $3 \mathrm{q}$ loci was excluded in two HSN1 families $(11,12)$, indicating the presence of at least a third gene.

The genetic defect linked to the 9q22 locus in the HSN1 families is associated with missense mutations in the SPTLC1 gene $(13,14)$. SPTLC1 encodes the long-chain base 1 (LCB1) subunit of the serine palmitoyltransferase (SPT) $(15,16)$. SPT catalyzes the condensation of L-serine and palmitoyl coenzyme A (CoA) to generate 3-ketodihydrosphingosine (KSD), the first committed step in de novo sphingolipid synthesis. The enzyme is ubiquitously expressed in various tissues in mammals, and activity is primarily associated with the endoplasmic reticulum $(17,18)$. Eukaryotic SPT is likely to be a heterodimer, in which LCB1 and LCB2 subunits are expressed by the SPTLC1 and SPTLC2 genes, respectively (19). 
Three mutations, C133Y, C133W, and V144D, have been identified in HSN1 patients $(13,14)$. These are located within a 12 -amino acid segment encoded by exons 5 and 6 of the SPTLC1 gene (our own unpublished data). Dawkins et al. (14) reported a higher synthesis of glucosylceramide (GlcCer) in cultured lymphocytes from HSN1 patients than in cell lines from healthy controls. Based on this observation, these authors suggested that these HSN1 mutations in the human LCB1 subunit were associated with an increase of SPT activity and represented gain-of-function mutations. However, the corresponding mutations in yeast have been shown recently to reduce SPT activity (20). Therefore, to begin to elucidate the mechanisms by which the mutations in the SPTLC1 gene cause HSN1, it is essential to clarify the impact of the mutations on the function of SPT in mammalian cells. In the present study we show that the two mutations in the LCB1 subunit that we have identified in our HSN1 families confer dominant negative effects on SPT activity in various cell types, including cultured lymphoblasts from HSN1 patients.

\section{Methods}

HSN1 families. Family D1 is American and of German origin. This family has been described previously $(2,8$, $21,22)$. In this family, the age of onset of the disease is usually in the second or third decade. The symptoms begin with subtle loss of pain and temperature perception and are followed by ulcers in the toes and fingers. Symptom onset is earlier and progresses faster in men than in women. Absence of a pinprick response is observed in the early teens, even in asymptomatic patients carrying the disease haplotype. Muscle weakness and atrophy are eventually evident in most patients and may be very severe, requiring mechanical assistance such as the use of splints and crutches. In the advanced cases, sensory potentials are absent, and motor nerve conduction velocities are decreased in the lower extremities (2). Most patients reported the sensation of shooting pain. Family N1 is Canadian and of English origin. In this family, the age and pattern of onset are equivalent to that in family D1. The sensory nerve action potentials are lost early, and the motor nerve conduction velocities are slower than expected from purely axonal lesions. Weakness of the hands and the legs also is evident, with shoulder pain and ulceration of the tip of the toes reported in later stages of the disease (23). In family D1, cysteine at position 133 of the coding region of SPTLC1 is substituted to tyrosine (C133Y); in family N1, the same cysteine is substituted to tryptophan (C133W) (13).

Chemicals. Cell culture media, palmitoyl CoA, L-serine, and anti-FLAG M2 affinity gel were purchased from Sigma-Aldrich (St. Louis, Missouri, USA). Sucrose monolaurate was from Dojindo Laboratories (Kumamoto, Japan). L- $\left[{ }^{3} \mathrm{H}(\mathrm{G})\right]$ serine $(20 \mathrm{Ci} / \mathrm{mmol})$ was from Moravek Biochemicals (Brea, California, USA), and $\mathrm{L}-\left[{ }^{14} \mathrm{C}(\mathrm{U})\right]$ serine $(157 \mathrm{mCi} / \mathrm{mmol})$ was from
Amersham Pharmacia Biotech (Piscataway, New Jersey, USA). TLC plates were from Merck KGaA (Darmstadt, Germany). Cell culture reagents were purchased from Invitrogen Corp. (Carlsbad, California, USA).

Plasmids. pSV-cLCB and pSV-FHcLCB1 are mammalian expression plasmids for wild-type hamster LCB1 subunit (24) and its FLAG/hexahistidine-tagged version (19), respectively. HSN1-like mutations were introduced to pSV-cLCB1 and pSV-FHcLCB1 by oligonucleotide-directed site-specific mutagenesis with the SP- 6 primer as the forward primer and appropriate mutation primers (5'-AAATCCTCGAGGTC CATAGGT ACCCAC-3' $3^{\prime}$ and $5^{\prime}$-AAATCCTCGAGGTCCCCAGGTACCCAC $-3^{\prime}$ for $\mathrm{C} 133 \mathrm{Y}$ and $\mathrm{C} 133 \mathrm{~W}$, respectively; the boldfaced nucleotides correspond to the point mutations) as the reverse primers for PCR. The mutated PCR fragments were digested with SalI and XhoI, and the SalIXhoI fragments were ligated with a phosphatase-treated vector derived from pSV-cLCB1 digested with SalI and $X h o I$. The resulting plasmids are systematically referred to as pSV-cLCB1C133Y, pSV-cLCB1C133W, pSV-FHcLCB1C133Y, and pSV-FHcLCB1C133W. The nucleotide sequence of the construct was verified by using an automated DNA sequencer.

Cell culture and transfection of lymphoblastoid and Chinese hamster ovary cells. Informed consent was secured from patients and healthy volunteers before obtaining peripheral blood, following institutional guidelines under an approved human research protocol. Lymphocytes were isolated from peripheral blood and transfected with the Epstein-Barr virus to establish lymphoblast cultures. Lymphoblastoid cell lines used are listed in Table 1 . The transformed cells were cultured in suspension at $37^{\circ} \mathrm{C}$ and $5 \% \mathrm{CO}_{2}$ in Iscove's medium supplemented with L-glutamine, 5\% FBS, 100 $\mathrm{U} / \mathrm{ml}$ penicillin, and $100 \mu \mathrm{g} / \mathrm{ml}$ streptomycin. LY-B strain, a Chinese hamster ovary (CHO) cell mutant, was established previously by us (25). CHO cells were routinely cultured in Ham's F12 medium supplemented with $10 \%$ newborn calf serum, $100 \mathrm{U} / \mathrm{ml}$ penicillin $\mathrm{G}$, and $100 \mu \mathrm{g} / \mathrm{ml}$ streptomycin sulfate (F12/NCS medium) at $33^{\circ} \mathrm{C}$.

Transfection of $\mathrm{CHO}$ cells with plasmids was performed with Lipofectamine PLUS reagent (Invitrogen Corp.), as recommended by the manufacturer. In brief, subconfluent monolayers of $\mathrm{CHO}$ cells were incubated with the complex of DNA and the reagent in a serumfree medium at $37^{\circ} \mathrm{C}$ for 5 hours. The cells were first cultured in a medium supplemented with $10 \%$ newborn calf serum at $33^{\circ} \mathrm{C}$ for 16 hours, then reseeded and cultured in F12/NCS medium. For analysis of transiently transfected cells, cell lysate was prepared 48 hours after the start of transfection, unless otherwise noted. CHO-K1/cLCB, CHO-K1/cLCBC133Y, and CHO-K1/cLCBC133W strains, which stably overproduced the respective LCB1 types, were isolated from G418-resistant transfectants of $\mathrm{CHO}-\mathrm{K} 1$ cells with pSVcLCB1, pSV-cLCB1C133Y, and pSV-cLCB1C133W, respectively, by limiting dilution. 
Table 1

Origins of lymphoblast cell lines used

$\begin{array}{lcccc}\text { Cell line ID no. } & \text { HSN1 phenotype } & \text { Mutation } & \text { Age (years) } & \text { Gender } \\ 4513 & \text { Healthy control } & & 27 & \text { Male } \\ 4539 & \text { Affected } & \text { C133Y } & 28 & \text { Male } \\ 4547 & \text { Healthy control } & & 58 & \text { Male } \\ 4516 & \text { Affected } & \text { C133Y } & 47 & \text { Male } \\ 4523 & \text { Healthy control } & & 57 & \text { Female } \\ 4556 & \text { Affected } & \text { C133Y } & 46 & \text { Female } \\ 8629 & \text { Healthy control } & & 38 & \text { Male } \\ 8628 & \text { Affected } & \text { C133W } & 42 & \text { Male }\end{array}$

Metabolic labeling of lipids with serine in lymphoblastoid cells or $\mathrm{CHO}$ cells. Lymphoblastoid cells $\left(3.2 \times 10^{6}\right.$ cells $/ \mathrm{ml}$ ) were incubated in $8 \mathrm{ml}$ of Iscove's medium supplemented with L-glutamine, $1 \% \mathrm{FBS}$, and $20 \mu \mathrm{Ci}$ $\mathrm{L}-\left[{ }^{3} \mathrm{H}(\mathrm{G})\right]$ serine at $37^{\circ} \mathrm{C}$ for 2.5 hours. Sphingolipids were separated by high-performance TLC as described previously (26). Radioactivity in each lipid fraction was determined using a scintillation counter. In $\mathrm{CHO}$ cells, analyses were performed as described previously $(24,27)$. Cells grown in $60-\mathrm{mm}$ dishes were incubated in $1.5 \mathrm{ml}$ of a serum-free Nutridoma medium containing $0.75 \mu \mathrm{Ci} \mathrm{L}-\left[{ }^{14} \mathrm{C}(\mathrm{U})\right]$ serine at $37^{\circ} \mathrm{C}$ for 2 hours. Lipids extracted from the labeled cells were separated on TLC plates with a solvent of methyl acetate/ $n$-propanol/chloroform/methanol $/ 0.25 \% \mathrm{KCl}$ (25/25/25/10/9 vol/vol). Radioisotope-containing lipids were visualized on TLC plates by autoradiography, and the intensity of the signals in each lipid fraction was determined using a BAS2000 image analyzer (Fuji Film Co., Tokyo, Japan). The rates of the lipid labeling were normalized for protein.

Preparation of microsomes. Microsomes from lymphoblastoid cell lines were prepared as described (17), with slight modification. Briefly, after three washes in PBS, cells were homogenized with a Teflon-glass homogenizer in $50 \mathrm{mM}$ HEPES-NaOH buffer $(\mathrm{pH}$ 7.4) containing $250 \mathrm{mM}$ sucrose and $5 \mathrm{mM}$ EDTA and disrupted by sonication at medium speed $(2 \times 10$ seconds) while on ice, then centrifuged at $10,000 \mathrm{~g}$ for 30 minutes. To maximize the yield of microsomes, the resulting pellet was homogenized and centrifuged for an additional 20 minutes, and this step was repeated twice. Then the combined supernatant was sedimented, at $10^{5} \mathrm{~g}$ for 60 minutes. The microsome-enriched pellet was washed in 50 mM HEPES$\mathrm{NaOH}$ buffer ( $\mathrm{pH}$ 7.4) containing 5 mM EDTA, 5 $\mathrm{mM} \mathrm{DTT}$, and $20 \%(\mathrm{wt} / \mathrm{vol})$ glycerol and dispersed in the same buffer $(0.25 \%$ vol of the cell culture). Lysate and microsomes of $\mathrm{CHO}$ cells were prepared as described previously (28), with minor modification. Briefly, CHO cells were disrupted in buffer L $(10 \mathrm{mM}$ HEPES-NaOH buffer, pH 7.5, containing $250 \mathrm{mM}$ sucrose, $1 \mathrm{mM}$ EDTA, and a protease inhibitor cocktail, Complete Protease Inhibitor; Roche Molecular Biochemicals, Mannheim, Germany) by sonication. For microsome preparation, after centrifugation
(2000 g, 15 minutes) of the cell lysate, the supernatant was subjected to high-speed centrifugation $\left(10^{5} \mathrm{~g}, 1\right.$ hour $)$, and the microsome-precipitate was suspended in buffer $\mathrm{L}$ and stored at $-80^{\circ} \mathrm{C}$ until use.

Assay of SPT activity. SPT assay was performed as described previously $(29,30)$. In brief, microsomes (100 $\mu \mathrm{g}$ protein) of lymphoblasts or lysate $(100 \mu \mathrm{g}$ protein $)$ of CHO cells were incubated in $0.2 \mathrm{ml}$ of HEPES$\mathrm{NaOH}$ buffer $(100 \mathrm{mM}, \mathrm{pH} 8.3$, for lymphoblast and 50 $\mathrm{mM}, \mathrm{pH}$ 7.5, for CHO cells) containing $5 \mathrm{mM}$ DTT, 2.5 mM EDTA, $50 \mu \mathrm{M}$ pyridoxal phosphate (PLP), $0.2 \mathrm{mM}$ palmitoyl $\mathrm{CoA}$, and $\mathrm{L}-\left[{ }^{3} \mathrm{H}(\mathrm{G})\right]$ serine $(1 \mathrm{mM}$ of 20 $\mathrm{mCi} / \mathrm{mmol}$ for lymphoblast and $0.1 \mathrm{mM}$ of 50 $\mathrm{mCi} / \mathrm{mmol}$ for $\mathrm{CHO}$ cells). A negative control reaction was included for each assay in which palmitoyl CoA was omitted. After incubation at $37^{\circ} \mathrm{C}$ for 10 minutes, the $\left[{ }^{3} \mathrm{H}\right]-\mathrm{KSD}$ formed was determined.

SDS-PAGE and Western immunoblot analysis. Microsomes prepared from human lymphoblast cells were subjected to $4-15 \%$ (wt/vol) gradient PAGE using the buffer system of Laemmli and electrophoretically blotted to PVDF membranes. Lysate and microsomes prepared from $\mathrm{CHO}$ cells were subjected to $10 \%$ (wt/vol) PAGE and blotted to the membranes. After blocking in saline containing $10 \%$ (wt/vol) nonfat dry milk and $0.1 \%$ Tween-20, the blot was incubated with primary Ab's, washed with saline containing $0.1 \%$ Tween-20, and incubated with horseradish peroxidase-conjugated secondary Ab. After several washes with saline containing $0.1 \%$ Tween-20, SPT subunits were detected using an enhanced chemiluminescence detection system (Amersham Pharmacia Biotech). An $\mathrm{mAb}$ directed against mouse LCB1 protein (Transduction Laboratories, Lexington, Kentucky, USA) was used for the detection of human LCB1, and antimLCB2 $\mathrm{Ab}$ raised against a glutathione transferase-mouse LCB2 fusion protein (16) (a gift from B. Weiss, University of Cologne, Cologne, Germany) was used for the detection of human LCB2. Anti-cLCB1 $73 / 90$ and anti-cLCB2 26/45, which are polyclonal $A b$ 's directed against synthetic peptides of hamster LCB1 and LCB2, respectively $(19,25)$, were used as the primary Ab's for detection of hamster LCB1 subunit and LCB2 subunit, respectively.

Coimmunoprecipitation of the LCB2 subunit with FLAGtagged LCB1 subunits. CHO-K1 cells grown to subconfluence in $100-\mathrm{mm}$ dishes were transfected with $2 \mu \mathrm{g}$ of various plasmids. The transfected cells were reseeded and cultured in F12/NCS medium at $33^{\circ} \mathrm{C}$ for an additional 3 days. The cells were harvested by scraping, and cell membranes were prepared as described above. Membranes (120 $\mu \mathrm{g}$ protein) were solubilized with $1 \%$ (wt/vol) sucrose monolaurate in $400 \mu \mathrm{l}$ of buffer $\mathrm{A}$ (100 mM sodium phosphate buffer, $\mathrm{pH}$ 8.0, containing $300 \mathrm{mM} \mathrm{NaCl}$ and $100 \mathrm{mM}$ sucrose). Note that SPT can be efficiently solubilized without inactivation under the conditions we used $(19,25)$. After centrifugation $\left(10^{5} \mathrm{~g}, 30\right.$ minutes), solubilized supernatant fluid was incubated with anti-FLAG M2 affinity gel 


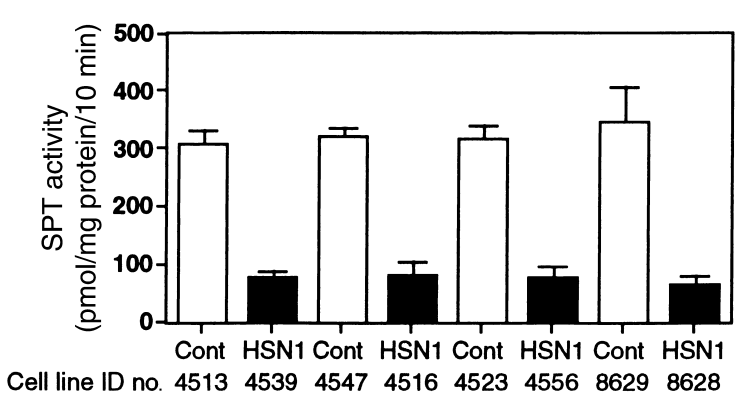

Figure 1

Comparison of SPT-specific activity between HSN1 patients and healthy controls. SPT activity was determined in microsomes isolated from lymphoblastoid cell lines of HSN1 patients (HSN1) and ageand gender-matched healthy controls (Cont). Each assay was performed in triplicate, and data are presented as means with SD. Cell line ID numbers correspond to those listed in Table 1.

( $25 \mu \mathrm{l}$ bed volume) equilibrated with buffer A containing $0.1 \%$ sucrose monolaurate for 1 hour. After precipitation of the resin $\left(10^{4} \mathrm{~g}, 30\right.$ seconds), pelleted gel was washed three times with $1 \mathrm{ml}$ of buffer A containing $0.1 \%$ sucrose monolaurate. Then, the washed gel was incubated in $100 \mu \mathrm{l}$ of the SDS-sample buffer containing $50 \mathrm{mM}$ DTT in place of $2 \%$ (wt/vol) 2-mercaptoethanol at $70^{\circ} \mathrm{C}$ for 5 minutes. After precipitation of the gel, the supernatant fluid was subjected to Western blot analysis of the LCB2 subunit.

Determination of protein concentrations. Protein concentrations of the preparations were determined using the Lowry method (31), with BSA as the standard.

\section{Results}

SPT-specific activity is decreased in HSN1 patients. We established virally transformed lymphoblastoid cell lines from four HSN1 patients and their age- and gendermatched healthy control individuals (Table 1). The control individuals are unrelated family members in which mutations in the SPTLC1 gene were excluded by genotype analysis. To determine whether mutations in the SPTLC1 gene result in alterations in enzyme activity, we measured SPT activity in microsomal preparations isolated from the cultured lymphoblasts. We performed the in vitro enzyme assay under optimal conditions and found SPT activity to be linear with protein concentrations (data not shown). HSN1 lymphoblasts exhibited greater than 50\% reduction in SPT activity (on a per milligram microsomal protein basis) compared with that in the lymphoblast cells from healthy individuals (Figure 1).

HSN1 patients express normal levels of $L C B 1$ and $L C B 2$ proteins. To determine whether the reduction in SPT activity is caused by a decrease of protein subunit levels, we compared the levels of LCB1 and LCB2 in microsomal preparations isolated from HSN1 lymphoblasts with those from controls (Figure 2). The microsomal preparations were from the same HSN1 patients and healthy controls as for the enzyme assay. In agreement with the predicted sizes of SPT subunits and with values obtained from HEK 293 human embryonic kidney cells (16) and human cultured keratinocytes (32), we detected a protein band of approximately $53 \mathrm{kDa}$ with two anti-LCB1 Ab's, i.e., a polyclonal $\mathrm{Ab}(\mathrm{Ab} 223 / 236)$ directed against a synthetic peptide of hamster LCB1 (Figure 2, upper panel) and a mAb directed against mouse LCB1 (data not shown). Using an anti-mouse $\mathrm{Ab}$ raised against a GST-mLCB2 fusion protein, we detected a slightly larger protein band than expected $(63 \mathrm{kDa})$ (Figure 2, lower panel). No differences in LCB1 or LCB2 protein levels were detected between HSN1 patients and healthy controls. In the LCB2 immunoblot, a faint band that appeared slightly larger than the predominant form of the LCB2 protein was also detected (Figure 2, lower panel). Although it remains unknown whether this faint band represents a modified form of the LCB2, there was no significant difference in the level of this band between HSN1 cells and controls. These results exclude either the repression of LCB mRNA transcription or inhibition of LCB mRNA translation as the primary mechanism(s) for reduced SPT activity in these HSN1 patients. In addition, these data demonstrate that the LCB1 mutations did not interfere with the stability of the translated proteins. Instead, the SPT-LCB1 mutations may directly interfere with the function of the enzyme.

A decrease of sphingolipid synthesis in lymphoblasts from HSN1 patients. To examine whether the HSN1 mutations affected de novo synthesis of sphingolipids, we performed metabolic labeling of lipids with radioactive serine in cultured lymphoblasts. For this we used six cell lines, three from patients and three from healthy controls. Labeled total sphingolipids, the sum of sphingomyelin (SM), GlcCer, and ceramide (Cer) were decreased (43\%) in lymphoblasts from HSN1 patients, compared with that of lymphoblasts from healthy controls (Figure 3). As for individual sphingolipids, synthesis levels of SM and Cer in HSN1 cell lines were significantly reduced. In contrast, labeling of phosphatidylserine (PS) was not significantly reduced in HSN1 lymphoblasts, compared

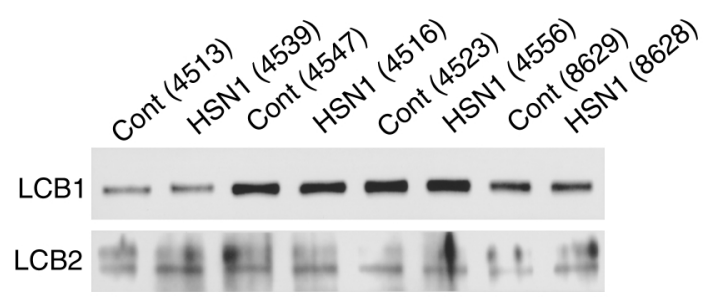

Figure 2

Western immunoblot analysis of LCB1 and LCB2 proteins present in lymphoblastoid cells from HSN1 patients and healthy controls. Each lane contains an equal amount $(5 \mu \mathrm{g})$ of microsomal proteins isolated from lymphoblastoid cells of healthy controls (Cont) and from HSN1 patients (HSN1). LCB1 and LCB2 proteins were detected by Western immunoblot analysis as described in Methods. The ID numbers of cell lines used are in parentheses. 


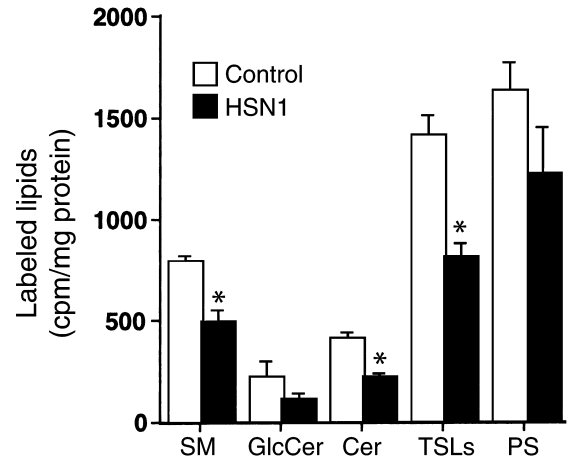

Figure 3

Metabolic labeling of lipids with radioactive serine in lymphoblastoid cells from HSN1 and healthy controls. Cells were incubated with $\mathrm{L}-\left[{ }^{3} \mathrm{H}(\mathrm{G})\right]$ serine for 2.5 hours. Lipids were isolated by high-performance TLC as described in Methods. Lipid synthesis was expressed as counts per minute per milligram of protein. The mean values with SD from triplicate experiments are shown. ${ }^{*} P<0.01 \mathrm{vs}$. the healthy control levels. TSLs, total sphingolipids.

with that of the control (Figure 3). These results were in agreement with the lower SPT activity in lymphoblasts from HSN1 patients.

The LCB1 subunits with HSN1-like mutations are unable to rescue $\mathrm{CHO}$ mutant cells lacking the endogenous LCB1 subunit. To further elucidate the mechanistic consequences of the LCB1 mutations, we next used an SPT-defective CHO cell mutant strain (LY-B) that lacks the endogenous LCB1 subunit (25). In this strain, the SPT activity deficit is specifically complemented by the wild-type LCB1 subunit (24). To examine whether the LCB1 subunit with the HSN1 mutations is capable of complementing the defect of the LY-B strain, we generated recombinant plasmids for expression of the wild-type hamster LCB1 subunit and its C133Y or C133W mutants. These plasmids were then transfected into
LY-B cells. Under transient expression conditions, the wild-type and mutant LCB1 subunits were similarly overproduced to approximately tenfold the endogenous LCB1 level observed in normal CHO-K1 cells (Figure $4 \mathrm{a}$, lane 1 vs. lanes 4-6). Transfection with the empty vector did not affect the lack of the LCB1 subunit in LY-B cells (Figure 4a, lane 2 vs. lane 3). Metabolic studies using radiolabeled serine revealed that de novo sphingolipid synthesis in LY-B cells was restored by expression of the wild-type LCB1 subunit, but not by expression of either mutant subunit (Figure 4b). Consistently, SPT activity in wild-type LCB1-transfected LY-B cells was restored to approximately $60 \%$ of the level in wild-type $\mathrm{CHO}$ cells, while the activity in mutant cLCB1-transfected LY-B cells was less than 10\% of the level in wild-type CHO cells (Figure 4c). Transfection efficiency under the conditions used was estimated to be approximately $60 \%$ when assessed using a reporter plasmid encoding a green fluorescent protein (data not shown). These results indicate that the $\mathrm{C} 133 \mathrm{Y}$ and C133W mutations impair the ability of the LCB1 subunit to support SPT enzymatic activity.

Expression of HSN1 LCB1 subunit variants of LCB1 subunit represses SPT activity in wild-type CHO-K1 cells. To examine whether expression of the mutated LCB1 subunits can affect SPT activity in wild-type $\mathrm{CHO}$ cells, we isolated stable transfectants of the CHO-K1 strain that overproduced either wild-type cLCB1 or mutant cLCB1 (i.e., cLCB1C133Y or CLCB1C133W) subunits to five- to tenfold the endogenous level (Figure 5a). The three stable transfectants are hereafter referred to as CHO-K1/cLCB1, CHO-K1/cLCB1C133Y, and CHO-K1/cLCB1C133W. Interestingly, SPT activity in CHO-K1/cLCB1C133Y and CHO-K1/cLCB1C133W attained only about $40 \%$ and $20 \%$ of the levels in control, untransfected CHO-K1 cells, whereas the activity in CHO-K1/cLCB1 was slightly (approximately 20\%)

\section{Figure 4}

Expression of wild-type hamster LCB1 and its C133Y or C133W mutant in LY-B cells. LY-B cells were transfected with various plasmids and analyzed. As controls, untransfected LY-B and wild-type CHO-K1 cells were also analyzed. Lanes 1 and 2 represent untransfected $\mathrm{CHO}-\mathrm{K} 1$ and LY-B cells, respectively. Lanes 3, 4, 5, and 6 represent LY-B cells transfected with pSV-OK (empty vector), pSV-cLCB1, pSV-cLCB1C133Y, and pSV-cLCB1C133W, respectively. (a) Western blotting of the LCB1 subunit. Each lane was loaded with $20 \mu \mathrm{g}$ protein of cell lysate. (b) Metabolic labeling of lipids. After incubation of cells with $\mathrm{L}-\left[{ }^{14} \mathrm{C}(\mathrm{U})\right]$ serine for 2 hours, lipids were extracted from the cells and separated by TLC. The radioactive image of lipids on the TLC plate is shown. PE, phosphatidylethanolamine. (c) SPT activity in cell lysate. Data shown are from triplicate experiments. a

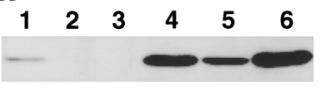

1. $\mathrm{CHO}-\mathrm{K} 1$

2. $\mathrm{LY}-\mathrm{B}$

3. LY-B + pSV-OK

4. $L Y-B+p S V-c L C B 1$

5. LY-B + pSV-cLCB1C133Y

6. LY-B + pSV-cLCB1C133W
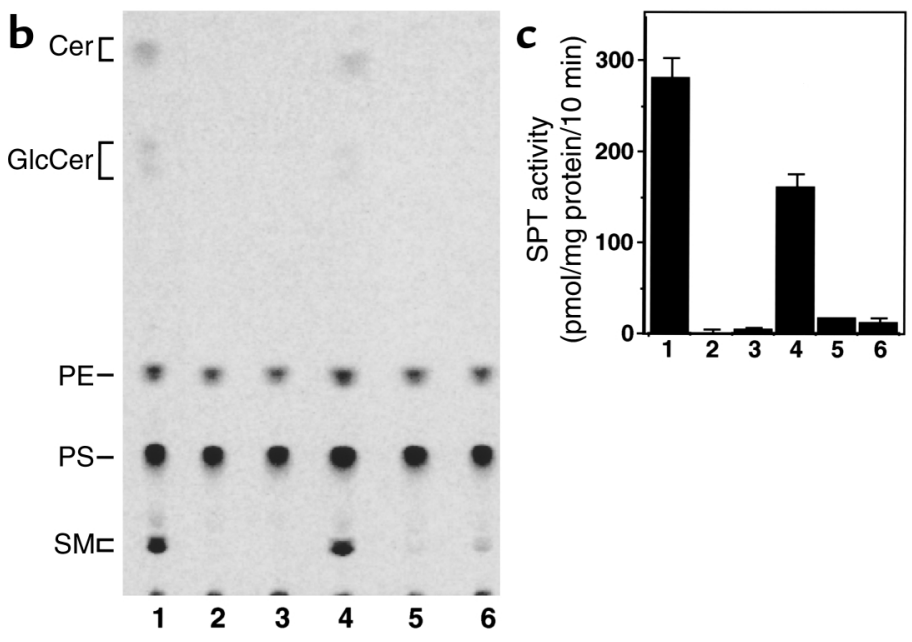
$\mathbf{a}$

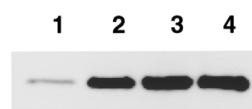

1. $\mathrm{CHO}-\mathrm{K} 1$

2. CHO-K1/CLCB1

3. CHO-K1/cLCB1C133Y

4. CHO-K1/cLCB1C133W

b

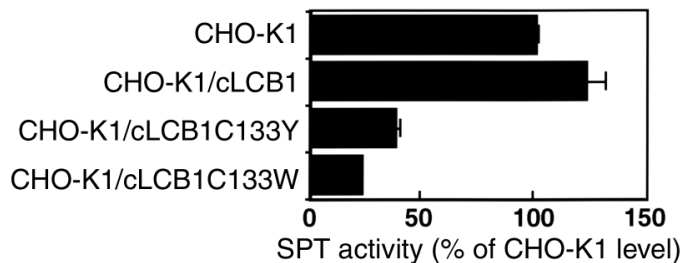

C

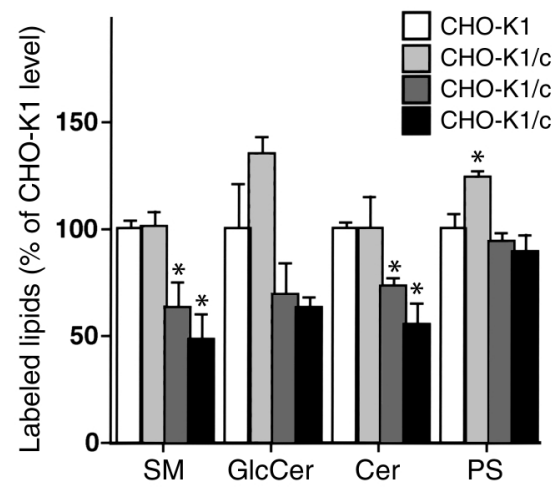

Figure 5

Stable overexpression of wild-type hamster LCB1 and its C133Y or C133W mutant in CHO-K1 cells. (a) Western blotting for the LCB1 subunit. Each lane was loaded with $20 \mu \mathrm{g}$ protein of cell lysate. (b) SPT activity in cell lysate. Data shown are from triplicate experiments. (c) Metabolic labeling of lipids in intact cells with $\mathrm{L}-\left[{ }^{14} \mathrm{C}(\mathrm{U})\right]$ serine. Radioactivity of each lipid type was determined and normalized for protein. Data are shown as the percentage of the mean values obtained in $\mathrm{CHO}-\mathrm{K} 1$ cells from four experiments. ${ }^{*} P<0.01$ vs. the $\mathrm{CHO}-\mathrm{K} 1$ levels.

higher than the control level (Figure 5b). Moreover, metabolic labeling experiments with radioactive serine showed that the rates of de novo synthesis of SM and Cer were significantly reduced in CHO-K1/ cLCB1C133Y and CHO-K1/cLCB1C133W cells, but unaltered in CHO-K1/cLCB1 cells, compared with the untransfected control rates (Figure 5c). The rate of PS synthesis was not significantly affected by expression of the mutant LCB1 products (Figure 5c), ruling out the possibility of nonspecific inhibition of lipid metabolism and/or serine uptake in CHO-K1/ cLCB1C133Y and CHO-K1/cLCB1C133W cells. These results indicate that the HSN1-equivalent CLCB1 mutants, when expressed in wild-type CHO cells, dominantly inactivate both SPT activity and sphingolipid synthesis.

The mutated LCB1 subunits interact with the LCB2 subunit. In eukaryotes, the LCB1 subunit is associated with the LCB2 subunit to form the active SPT enzyme $(20,25)$. To determine if the HSN1-equivalent mutant LCB1 proteins interact with the LCB2 subunit, we expressed both normal and mutant FLAG-tagged cLCB1 proteins in CHO-K1 cells and performed immunoprecipitation with an anti-FLAG Ab using the solubilized membrane fraction from the cells. The endogenous LCB2 subunit was coimmunoprecipitated, with both the normal FLAG-tagged CLCB1 protein and the FLAG-tagged cLCB1C133Y and CLCB1C133W proteins (Figure 6). The possibility of nonspecific precipitation of the LCB2 subunit was eliminated, because no LCB2 subunit was detected in the immunoprecipitated fraction from the cells transfected with nontagged cLCB1 constructs (Figure 6). Therefore, these results demonstrate that the wild-type LCB1 and both cLCB1C133Y and cLCB1C133W mutant proteins form a complex with the LCB2 subunit, strongly suggesting that direct protein-protein interactions cause the diminished activity in HSN1 cells.

\section{Discussion}

In the present study we have clarified some of the key molecular aspects of mammalian SPT activity and have defined how the HSN1-associated LCB1 subunit mutations lead to diminished enzyme activity. We demonstrated that the C133Y and C133W mutations in the SPTLC1 gene result in decreased SPT activity in lymphoblasts from HSN1 patients. In addition, these mutations not only impaired the ability of the LCB1 subunit to support SPT activity in a CHO cell line that lacks the endogenous LCB1 subunit, but also reduced SPT activity when expressed in the wild-type $\mathrm{CHO}$ strain. Based on these results, we conclude that both HSN1-LCB1 mutations alter SPT activity through a dominant negative effect, explaining why this form of HSN1 is dominantly inherited. Moreover, we demonstrated in normal CHO cells that both mutant LCB1 proteins interact with the wild-type LCB2 subunit. Accordingly, we suggest that the competition between the mutated and wild-type LCB1 for interaction with LCB2 and formation of the SPT complex may account for the dominant negative effects of the mutations. The present results are consistent with the earlier observations showing that in yeast, HSN1-like mutations reduce SPT activity through a dominant negative effect (20).

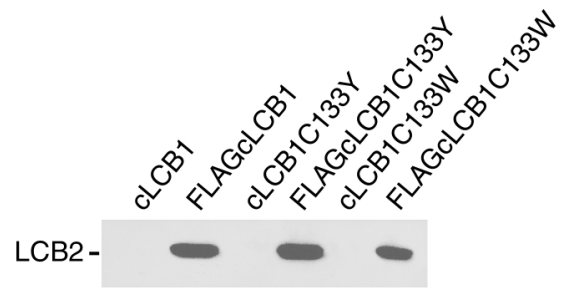

\section{Figure 6}

Coimmunoprecipitation of the LCB2 subunit with the LCB1 subunit and its HSN1-like mutants. After transfection of $\mathrm{CHO}-\mathrm{K} 1$ cells with expression plasmids encoding wild-type and mutated CLCB1 with or without the amino-terminal FLAG-tag, cell membranes were prepared. The membranes were solubilized with $1 \%$ (wt/vol) sucrose monolaurate, and the solubilized fraction was subjected to an antiFLAG Ab-coupled matrix. After washing of the matrix, proteins bound to the matrix were eluted by incubation in the SDS-sample buffer at $70^{\circ} \mathrm{C}$ for 5 minutes, and analyzed by Western blotting with anti-CLCB2 Ab as described in Methods. 
However, our results differ from a previous study in which, in HSN1 lymphoblastoid cells, the SPTLC1 mutations resulted in increased levels of GlcCer (14). There might be a possibility that reduction of SPT activity in HSN1 lymphoblasts is due simply to a genedosage effect of wild-type SPTLC1 rather than to the dominant negative effects of the mutated alleles, although overproduction of mutated $L C B 1$ products clearly gave negative effects on SPT activity in $\mathrm{CHO}$ cells. When one copy of yeast $L C B 1$ having HSN1-like mutations is introduced to haploidal $L C B 1^{+}$yeast cells, however, SPT activity is reduced by approximately $50 \%$, whereas introduction of one copy of the wild-type LCB1 gene does not affect the activity (20). These results can be produced by dominant negative effects of the HSN1 mutations, but not by a gene-dosage effect of wild-type LCB1. Moreover, three SPTLC1 mutations have been detected in a total of 13 HSN1 families $(13,14)$, all of which are missense mutations. No deletions or nonsense mutations have been detected. Thus, HSN1mutated SPTLC1 alleles probably provide dominant negative effects on SPT activity in patient-derived cells, although a reduced dosage of wild-type SPTLC1 might also contribute to the reduction of SPT activity. Further evaluation of the dominant negative versus haploinsufficiency mechanisms in dominantly inherited HSN1 should await analysis with the as yet unavailable LCB1 heterozygous knockout mouse model.

SPT belongs to a group of PLP-dependent $\alpha$-oxoamine synthases (POASs) that catalyze the condensations of amino acids with carboxylic acid-CoA thioesters to generate $\alpha$-oxoamines. In addition to the similarities of the chemical reactions catalyzed, members of the POAS enzyme family have strong amino acid sequence similarities. The three-dimensional structure of 8-amino-7oxononanoate synthase (AONS) (33), a member of the POAS family, has yielded important insights into other POAS members. For example, the PLP cofactor binds to the enzyme in a cleft between the two AONS subunits, and residues from both subunits participate in the cofactor binding $(33,34)$. Although the crystal structure of SPT is not yet available, the AONS structure would suggest that the active site of SPT is composed of adjacent residues from both LCB1 and LCB2 subunits. By analogy, in addition to the amino acid residues in the LCB2 subunit that contribute to the structure of the SPT catalytic site, including the PLP-binding lysine residue, several specific residues of the LCB1 subunit probably contribute, as well. In this scenario, the mutant LCB1C133Y and LCB1C133W subunits are perhaps unable either to contribute to the formation of the active catalytic site or to alter the active site structure to interfere with enzyme activity. Consistent with this hypothesis, the sequence motif around Cys133 in LCB1 is highly conserved from yeast to human, suggesting that this region plays a crucial role in SPT structure and/or catalytic activity. In addition, when a tertiary structure of the yeast LCB1/LCB2 heterodimer complex is modeled according to the AONS structure, Cys 180 of yeast Lcb1p (which corresponds to
Cys 133 in mammalian LCB1 protein) resides at the interface between the two subunits in close proximity to the PLP-binding lysine residue of the Lcb2p (20).

Based upon the critical role of sphingolipids in the skin $(35,36)$, it is interesting to note that skin wounds accompany the approximate onset of sensory neuropathy. Whether damage to sensory neurons represents an initiating or contributing factor in HSN1 disease progression is unknown. However, preliminary results from a single patient did not reveal significant changes in epidermal Cer content or composition, suggesting that sufficient sphingolipid production occurs in this disease despite the HSN1 LCB1 mutation(s). Cutaneous lipid production, barrier function, and disease progression are being studied.

It remains unclear why mutations in a protein widely expressed in all tissues trigger pathology that is highly restricted to specific subsets of cells within a tissue. One explanation for this incongruity could be an as yet undefined alternative splice form of LCB1 and/or LCB2 in the dorsal root ganglia (DRG) on which the HSN1 mutations exert specific action. Alternatively, degeneration of DRG in HSN1 may be associated with one or more unique features of sensory neurons that reveal a critical sensitivity to the status of sphingolipids. For example, a slight reduction in sphingolipid production/content over an extended duration, due to a partial deficiency in SPT activity, could result in a selective damage of a subset of sensory neurons, thereby inducing the slowly progressive and late-onset sensory neuropathy.

CHO mutant cells completely defective in SPT activity cannot grow in a sphingolipid-deficient medium $(25,37)$. This loss of viability is reconstituted upon addition of exogenous sphingolipids $(25,37)$. In addition, a complete loss of SPT activity also results in embryonic lethality in the fruit fly (38). Interestingly, when SPT deficiency is partial, mutant flies grow into adults with abnormalities in various external organs, but such abnormalities are rescued upon feeding with sphingosine (38). Thus, our finding that the HSN1 mutations negatively affect the activity of mammalian SPT might indicate the possibility that external supply of sphingolipids would prevent or delay the appearance of clinical symptoms in patients affected by the HSN1 mutations.

Neurodegeneration in autosomal dominant diseases has been often associated with the accumulation of insoluble protein aggregates. For example, amyloid- $\beta$ and $\alpha$-synuclein have been shown to aggregate in Alzheimer disease $(39,40)$ and Parkinson disease (41), respectively. By analogy, it is conceivable that in addition to its dominant negative effect on SPT activity, mutant LCB1 might accumulate, introducing a new toxic function into neurons. Clearly, neuronal cell models and animal models of HSN1 will help answer these and other questions related to the pathogenic mechanism(s) of HSN1 and the development of possible prophylactic methods. 


\section{Acknowledgments}

We thank the patients and family members that we studied for their time, patience, and commitment. We thank Tomoko Hara for her excellent technical assistance. This work was supported by grants from the Muscular Dystrophy Association Inc., the Deater Foundation Inc., the Neuropathy Association Inc., and the Ministry of Education, Culture, Sports, Science and Technology of Japan.

1. Denny-Brown, D. 1951. Hereditary sensory radicular neuropathy. Jour nal of Neurology, Neurosurgery, and Psychiatry. 14:237-252.

2. Whitaker, J.N., Falchuck, Z.M., Engel, W.K., Blaese, R.M., and Strober, W. 1974. Hereditary sensory neuropathy. Association with increased synthesis of immunoglobulin A. Arch. Neurol. 30:359-371.

3. Dyck, P.J. 1993. Neuronal atrophy and degeneration predominantly affecting peripheral sensory and autonomic neuron. In Peripheral new ropathies. P.J. Dyck, P.K. Thomas, J.W. Griffin, P.A. Low, and J.F. Poduslo, editors. W.B. Saunders Co., Philadelphia, Pennsylvania, USA. 1065-1093.

4. Hicks, E.P. 1922. Hereditary perforating ulcers of the feet. Lancet. 1:319-321.

5. Hageman, G., Hilhorst, B.G., and Rozeboom, A.R. 1992. Is there involvement of the central nervous system in hereditary sensory radicular neuropathy? Clin. Neurol. Neurosurg. 94:49-54.

6. Wallace, D.C. 1965. Observations upon a predominantly sensory hereditary neuropathy. Proc. Aust. Assoc. Neurol. 3:101-109.

7. Nicholson, G.A., et al. 1996. The gene for hereditary sensory neuropathy type I (HSN-I) maps to chromosome 9q22.1-q22.3. Nat. Genet. 13:101-104. 8. Bejaoui, K., et al. 1999. Confirmation of linkage of type 1 hereditary sensory neuropathy to human chromosome 9q22. Neurology. 52:510-515.

9. Dubourg, O., et al. 2000. Phenotypic and genetic study of a family with hereditary sensory neuropathy and prominent weakness. Muscle Nerve. 23:1508-1514

10. Kwon, J.M., Elliott, J.L., and Yee, W.C. 1995. Assignment of a second Charcot-Marie-Tooth type II locus to chromosome 3q. Am. J. Hum. Genet. 57:853-858.

11. Auer-Grumbach, M., Wagner, K., Timmerman, V., De Jonghe, P., and Hurtung, H.P. 2000. Ulcero-mutilating neuropathy in an Austrian kinship without linkage to hereditary motor and sensory neuropathy IIB and hereditary sensory neuropathy I loci. Neurology. 54:45-52.

12. Stogbauer, F., et al. 1999. Autosomal dominant burning feet syndrome. J. Neurol. Neurosurg. Psychiatry. 67:78-81.

13. Bejaoui, K., et al. 2001. SPTLC1 is mutated in hereditary sensory neuropathy, type 1. Nat. Genet. 27:261-262.

14. Dawkins, J.L., Hulme, D.J., Brahmbhatt, S.B., Auer-Grumbach, M., and Nicholson, G.A. 2001. Mutations in SPTLC1, encoding serine palmitoyltransferase, long chain base subunit-1, cause hereditary sensory neuropathy type I. Nat. Genet. 27:309-312.

15. Nagiec, M.M., Baltisberger, J.A., Wells, G.B., Lester, R.L., and Dickson, R.C. 1994. The LCB2 gene of Saccharomyces and the related LCB1 gene encode subunits of serine palmitoyltransferase, the initial enzyme in sphingolipid synthesis. Proc. Natl. Acad. Sci. USA. 91:7899-7902.

16. Weiss, B., and Stoffel, W. 1997. Human and murine serine-palmitoylCoA transferase-cloning, expression and characterization of the key enzyme in sphingolipid synthesis. Eur. J. Biochem. 249:239-247.

17. Holleran, W.M., Williams, M.L., Gao, W.N., and Elias, P.M. 1990. Serinepalmitoyl transferase activity in cultured human keratinocytes. J. Lipid Res. 31:1655-1661.

18. Mandon, E.C., Ehses, I., Rother, J., van Echten, G., and Sandhoff, K. 1992. Subcellular localization and membrane topology of serine palmitoyltransferase, 3-dehydrosphinganine reductase, and sphinganine $\mathrm{N}$-acyltransferase in mouse liver. J. Biol. Chem. 267:11144-11148.

19. Hanada, K., Hara, T., and Nishijima, M. 2000. Purification of the serine palmitoyltransferase complex responsible for sphingoid base synthesis by using affinity peptide chromatography techniques. J. Biol. Chem. 275:8409-8415.
20. Gable, K., et al. 2002. Mutations in the yeast LCB1 and LCB2 genes, including those corresponding to the hereditary sensory neuropathy type I mutations, dominantly inactivate serine palmitoyltransferase. J. Biol. Chem. 277:10194-10200.

21. Tocantins, L.M., and Reimann, H.A. 1939. Perforating ulcers of feet, with osseous atrophy in a family with other evidences of dysgenesis (hare lip, cleft palate): in an instance of probable myelodysplsia. JAMA. 1939:2251-2255.

22. Reimann, H.A., McKechnie, W.G., and Stanisavljevic, S. 1958. Hereditary sensory radicular neuropathy and other defects in a large family. Am. J. Med. 25:573-579.

23. Shivji, Z.M., and Ashby, P. 1999. Sympathetic skin responses in hereditary sensory and autonomic neuropathy and familial amyloid neuropathy are different. Muscle Nerve. 22:1283-1286.

24. Hanada, K., et al. 1997. A mammalian homolog of the yeast LCB1 encodes a component of serine palmitoyltransferase, the enzyme cat alyzing the first step in sphingolipid synthesis. J. Biol. Chem. 272:32108-32114.

25. Hanada, K., et al. 1998. Mammalian cell mutants resistant to a sphingomyelin-directed cytolysin. Genetic and biochemical evidence for complex formation of the LCB1 protein with the LCB2 protein for serine palmitoyltransferase. J. Biol. Chem. 273:33787-33794.

26. Uchida, Y., et al. 2000. Epidermal sphingomyelins are precursors for selected stratum corneum ceramides. J. Lipid Res. 41:2071-2082.

27. Fukasawa, M., Nishijima, M., and Hanada, K. 1999. Genetic evidence for ATP-dependent endoplasmic reticulum-to-Golgi apparatus trafficking of ceramide for sphingomyelin synthesis in Chinese hamster ovary cells. J. Cell Biol. 144:673-685.

28. Hanada, K., and Pagano, R.E. 1995. A Chinese hamster ovary cell mutant defective in the non-endocytic uptake of fluorescent analogs of phosphatidylserine: isolation using a cytosol acidification protocol. J. Cell Biol. 128:793-804.

29. Williams, R.D., Nixon, D.W., and Merrill, A.H. 1984. Comparison of serine palmitoyltransferase in Morris hepatoma 7777 and rat liver. Cancer Res. 44:1918-1923.

30. Merrill, A.H., Jr. 1983. Characterization of serine palmitoyltransferase activity in Chinese hamster ovary cells. Biochim. Biophys. Acta. 754:284-291.

31. Lowry, O.H., Rosebrough, N.J., Farr, A.L., and Randall, R.J. 1951. Protein measurement with the folin phenol reagent. J. Biol. Chem. 193:265-275.

32. Farrell, A.M., et al. 1998. UVB irradiation up-regulates serine palmitoyltransferase in cultured human keratinocytes. J. Lipid Res. 39:2031-2038.

33. Alexeev, D., et al. 1998. The crystal structure of 8-amino-7-oxononanoate synthase: a bacterial PLP-dependent, acyl-CoA-condensing enzyme. J. Mol. Biol. 284:401-419.

34. Webster, S.P., et al. 2000. Mechanism of 8-amino-7-oxononanoate synthase: spectroscopic, kinetic, and crystallographic studies. Biochemistry. 39:516-528.

35. Holleran, W.M., et al. 1991. Sphingolipids are required for mammalian epidermal barrier function. Inhibition of sphingolipid synthesis delays barrier recovery after acute perturbation. J. Clin. Invest. 88:1338-1345.

36. Holleran, W.M., et al. 1991. Regulation of epidermal sphingolipid synthesis by permeability barrier function. J. Lipid Res. 32:1151-1158.

37. Hanada, K., et al. 1992. Sphingolipids are essential for the growth of Chinese hamster ovary cells. Restoration of the growth of a mutant defective in sphingoid base biosynthesis by exogenous sphingolipids. J. Biol. Chem. 267:23527-23533.

38. Adachi-Yamada, T., et al. 1999. De novo synthesis of sphingolipids is required for cell survival by down-regulating c-Jun $\mathrm{N}$-terminal kinase in Drosophila imaginal discs. Mol. Cell. Biol. 19:7276-7286.

39. Younkin, S.G. 1995. Evidence that A beta 42 is the real culprit in Alzheimer's disease. Ann. Neurol. 37:287-288.

40. Scheuner, D., et al. 1996. Secreted amyloid beta-protein similar to that in the senile plaques of Alzheimer's disease is increased in vivo by the presenilin 1 and 2 and APP mutations linked to familial Alzheimer's disease. Nat. Med. 2:864-870.

41. Conway, K.A., Harper, J.D., and Lansbury, P.T. 1998. Accelerated in vitro fibril formation by a mutant alpha-synuclein linked to early-onset Parkinson disease. Nat. Med. 4:1318-1820. 